\title{
Deformation of drops by outer eddies in turbulence
}

\author{
Alberto Vela-Martín ${ }^{1,2} \dagger$, Marc Avila ${ }^{1}$ \\ ${ }^{1}$ Centre of Applied Space Technology and Microgravity (ZARM) University of Bremen, 28359 \\ Bremen \\ ${ }^{2}$ School of Aeronautics, Universidad Politécnica de Madrid, 28040 Madrid, Spain
}

(Received xx; revised xx; accepted xx)

Drop deformation in fluid flows is investigated here as an exchange between the kinetic energy of the fluid and the surface energy of the drop. We show analytically that this energetic exchange is controlled only by the stretching (or compression) of the drop surface by the rate-of-strain tensor. This mechanism is analogous to the stretching of the vorticity field in turbulence. Leveraging the non-local nature of turbulence dynamics, we introduce a new decomposition that isolates the energetic exchange due to local dropinduced surface effects, from the non-local action of turbulent fluctuations. We perform direct numerical simulations of single inertial drops in isotropic turbulence and show that an important contribution to the increments of the surface energy arises from the non-local stretching of the fluid-fluid interface by eddies far from the drop surface (outer eddies). We report that this mechanism is dominant and independent of surface dynamics in a range of Weber numbers in which drop breakup occurs. These findings shed new light on drop deformation and breakup in turbulent flows, and open the venue for the improvement and simplification of breakup models.

Key words: turbulence, drop breakup, diffuse-interface methods

\section{Introduction}

Turbulent binary mixtures of inmiscible fluids are ubiquitous in natural phenomena and industrial applications. Their physical properties depend strongly on the structure of the disperse phase, in which the breakup of fluid particles - drops or bubbles - plays a fundamental role. Understanding and modelling turbulent breakup is essential to predict and control the dynamics of inmiscible mixtures, but this remains a challenge to theoretical and empirical approaches. The physical mechanisms that drive particle deformation and breakup are still poorly understood.

The first studies of drop and bubble breakup in turbulent flows date back to the pioneering work of Kolmogorov (1949) and Hinze (1955), who proposed the idea of the maximum stable diameter based on dimensional analysis. This quantity provides a reasonable estimate of the predominant fluid-particle size in turbulent mixtures, and has been extensively validated in experiments and numerical simulations (Hinze 1955;

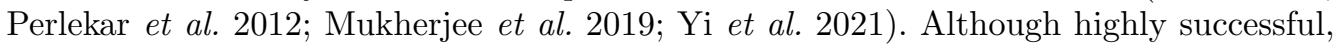
the maximum stable diameter does not provide information on the dynamics of breakup, which is essential to predict the spatio-temporal variations of fluid-particle distributions

$\dagger$ Email address for correspondence: albertovelam@gmail.com 
in complex flows (Hakansson 2019). The evolution of fluid-particle distributions are usually described with population balance equations (PBE), which use convolution kernels to model the effect of breakup and coalescence (for a recent review see Ramkrishna \& Singh 2014),. An important parameter of these kernels is the breakup rate, which depends on the particle size, the material properties of the mixture, and the characteristics of turbulence. A profusion of models predict the breakup rate based on different phenomenological assumptions (Lasheras et al. 2002, Liao \& Lucas 2009), but many of these assumptions are heuristic or cannot be readily tested against empirical data. As a consequence, these models provide contradictory results, and their ability to yield accurate predictions of particle-size distributions even in simple turbulent flows is limited (Aiyer et al. 2019). In addition, determining the breakup rate directly from experiments is not straightforward (Hakansson 2020), and data to validate and parameterise these models have been traditionally difficult to obtain. Laboratory experiments are challenging because of the difficulty to isolate surface tension effects from other effects such as buoyancy (Risso \& Fabre 1998), or large-scale anisotropies and inhomogeneities (Eastwood et al. 2004, Andersson \& Andersson 2006, Solsvik \& Jakobsen 2015), or to follow the evolution of single drops until breakup (Maaß \& Kraume 2012). Direct numerical simulations have recently filled this gap by producing high-quality data to study particle-turbulence interactions (Dodd \& Ferrante 2016) and particle breakup in turbulent emulsions (Perlekar et al. $\mid 2012$; Komrakova et al. | 2015, Scarbolo et al. 2015 , Roccon et al. |2017; Mukherjee et al.||2019), and in single drop simulations (Qian et al. 2006: Shao et al.| 2018; Zhong \& Komrakova 2019). A recent review on direct numerical simulations of particle-laden flows is Elghobashi (2019). Despite these advances, our limited understanding of the theoretical aspects of particle-turbulence interactions limits the exploitation of these results for breakup modelling.

Some commonly used breakup models rely on an energetic description of particle deformation and breakup (Coulaloglou \& Tavlarides 1976, Narsimhan et al. 1979, Luo \& Svendsen 1996). In this context, the surface energy of the particle is a marker of its degree of deformation, which increases due to interactions with turbulent fluctuations. This process is viewed as an energetic exchange between the turbulent kinetic energy of the flow and the surface energy of the particle. Similarly to the idea of the activation energy in chemistry, breakup is thought to occur when a threshold of the surface energy is reached (Andersson \& Andersson 2006). A widespread phenomenological picture describes the increments of the surface energy as caused by the 'impact' of eddies on the surface of the particle (see e.g. Lasheras et al. 2002; Liao \& Lucas 2009, for reviews). The kinetic energy of these eddies and their arrival frequency are relevant model parameters, and an efficiency factor is usually introduced to account for an incomplete energetic exchange in the process. The energetic approach to modelling breakup is convenient because it lumps the complexity of particle-turbulence interactions into model parameters, but it depends on the validity of the model assumptions about the energetic exchange between the surface energy and the kinetic energy of turbulent fluctuations.

An important limitation of the energetic approach is the lack of a quantitative framework to test and refine these phenomenological breakup models. In particular, the idea of 'impact' and the definition of eddies are fraught with ambiguity, and the connection of these concepts with measurable quantities in the flow is unclear. Recent studies have addressed the problem of the energetic exchange in fluid-fluid dispersions (Dodd \& Ferrante 2016, Rosti et al. 2019, Perlekar 2019, Mukherjee et al. 2019), but focusing mainly on global budgets, which limits their application to fluid-particle breakup.

This paper addresses the energetic problem of particle-turbulence interactions from a local perspective, and presents an analytical derivation of the local mechanism responsible 
for the energetic exchange between surface energy and kinetic energy. For our derivation, we use the Cahn-Hilliard-Navier-Stokes equations (Jacqmin 1999), which provide a thermodynamically consistent and convenient framework to study the interactions between turbulence and fluid-fluid interfaces from an energetic perspective. In these equations the interface has finite thickness, but in the limit of a sharp interface the classical stress balance at the infinitesimal fluid-fluid interface is recovered (Magaletti et al. 2013). We exploit this to show that the energetic exchange is solely described by the action of the rate-of-strain tensor on the surface of the fluid particle. The increments of the surface energy are a consequence of the stretching of the surface by the rate-of-strain tensor, a mechanism that resembles the stretching of the vorticity field in turbulence.

This result applies in general to fluid-fluid interfaces. Here we use it to study drop deformation in turbulence. We analyse data generated by direct numerical simulations of single drops embedded in homogeneous isotropic turbulence in range of Weber numbers in which breakup occurs. Using analytical tools borrowed from turbulence research (Ohkitani \& Kishiba 1995, Hamlington et al. 2008), we distinguish the self-induced increments due to surface (inner) dynamics from the action of the surrounding (outer) turbulence. We show that an important contribution to drop deformation and breakup stems from the nonlocal surface stretching by eddies away from the drop, which constitutes a quantitative reinterpretation of the phenomenological collision of eddies. This analysis provides a consistent quantitative framework to advance the theoretical understanding of breakup and its modelling.

This paper is organised as follows. In $\$ 2$, we present the derivation of the fundamental mechanisms that drives the exchange between the kinetic energy and the surface energy. We study this mechanism in direct numerical simulations of single drops, which are described in $\$ 3$. The results and their discussion are presented in $\$ 4$ and $\$ 5$, respectively. Finally, our main findings are summarised in $\$ 6$.

\section{The exchange between kinetic energy and surface energy}

We consider the Navier-Stokes (NS) equations coupled to the Cahn-Hilliard (CH) equation,

$$
\begin{aligned}
\rho\left(\partial_{t} u_{i}+u_{j} \partial_{j} u_{i}\right) & =-\partial_{i} \tilde{p}+2 \partial_{j} \mu S_{i j}+f_{i}-c \partial_{i} \phi, \\
\partial_{t} c+u_{j} \partial_{j} c & =\kappa \partial_{k k} \phi,
\end{aligned}
$$

which, together with the incompressibility constraint, $\partial_{i} u_{i}=0$, describe the evolution of an immiscible binary mixture of incompressible fluids (Jacqmin 1999). Here $u_{i}$ is the $i$-th component of the velocity vector, $\tilde{p}$ is a modified pressure, $S_{i j}=\frac{1}{2}\left(\partial_{i} u_{j}+\partial_{j} u_{i}\right)$ is the rate-of-strain tensor and $f_{i}$ is a body-force term per unit volume acting on the large scales to sustain turbulence. Repeated indices imply summation, and we consider periodic boundary conditions. The concentration of each component in the mixture is represented by $c$, where $c= \pm 1$ are the pure components. The density, $\rho$, and the dynamic viscosity, $\mu$, of the fluid mixture depend on $c$, and the immiscibility is modelled through a chemical potential,

$$
\phi=\beta\left(c^{2}-1\right) c-\alpha \partial_{k k} c
$$

The true pressure is related to the modified pressure by $p=\tilde{p}+c \phi-\beta / 4\left(c^{2}-1\right)^{2}+\alpha / 2\left(\partial_{i} c\right)^{2}$ (Jacqmin 1999). The action of interfacial forces in the momentum equation is represented by $c \partial_{i} \phi$, which is derived from physical energy-conservation arguments, and consistently reproduces the linear relation between surface tension forces and the local curvature of the interface (Jacqmin 1999). The numerical parameters $\alpha$ and $\beta$ determine the typical 
width of the fluid-fluid interface,

$$
\delta=4 \sqrt{2 \alpha / \beta}
$$

and the mobility, $\kappa$, determines its typical relaxation time. When these parameters are fixed appropriately, $\kappa \sim \delta^{2}$ (Magaletti et al. 2013), the interface is consistently close to the equilibrium profile, $c_{e q}(\xi)=\tanh (4 \xi / \delta)$, where $\xi$ is the spatial coordinate in the direction normal to the surface tangent plane. The surface tension reads

$$
\sigma=\alpha \int_{-\infty}^{+\infty}\left(\partial_{i} c_{e q}\right)^{2} \mathrm{~d} \xi=\frac{4}{3 \sqrt{2}} \sqrt{\alpha \beta} .
$$

The limits of the integral indicate that it is taken between large distances (formally infinite) compared to the interface thickness, $\delta$. In practice, $95 \%$ of the integral in 2.4 is contained in $\xi \in[-\delta / 2, \delta / 2]$.

\subsection{The evolution equations of the kinetic energy and the free energy}

We obtain the evolution equation of the kinetic energy of the flow by taking the dot product of the NS equations with $u_{i}$. For convenience, we use the transformation $-c \partial_{i} \phi=\phi \partial_{i} c-\partial_{i}(c \phi)$, and add the second term in the right-hand side to a new modified pressure, $p^{\prime}=\tilde{p}-c \phi$. Then the equation reads

$$
\partial_{t} e+u_{j} \partial_{j} e=\partial_{i}\left(-u_{i} p^{\prime}+2 \mu u_{j} S_{i j}\right)-2 \mu S_{i j} S_{i j}+u_{i} \phi \partial_{i} c+u_{i} f_{i},
$$

where $e=1 / 2 \rho u_{i} u_{i}$ is the turbulent kinetic energy per unit mass. The only terms contributing on average to the total kinetic energy budget are the local kinetic energy dissipation, $2 \mu S_{i j} S_{i j}$, the power input, $u_{i} f_{i}$, and the energetic exchange between the kinetic energy and the surface energy, $u_{i} \phi \partial_{i} c$.

By multiplying the $\mathrm{CH}$ equation by the chemical potential, we obtain

$$
\partial_{t} h+u_{j} \phi \partial_{j} c=\kappa \phi \partial_{k k} \phi
$$

where

$$
h=\beta / 4\left(c^{2}-1\right)^{2}+\alpha / 2\left(\partial_{k} c\right)^{2},
$$

is the free energy per unit volume. When integrated across the interface thickness, this quantity transforms into the energy per unit area of the interface,

$$
\sigma=\int_{-\infty}^{+\infty} h(\xi) \mathrm{d} \xi
$$

where we have assumed an equilibrium profile of the phase field, $c_{\text {eq }}$, and the integral is performed as in 2.4.

We now transform (2.6) into an advection equation for $h$ by decomposing the product $\phi \partial_{i} c$ and operating on the partial derivatives. We find the relation

$$
\phi \partial_{i} c=\partial_{i} h-\alpha \partial_{j} \tau_{i j},
$$

where $\tau_{i j}=\partial_{i} c \partial_{j} c$ is a Korteweg stress tensor. Substituting this expression into the kinetic energy equation and the free energy equation, we obtain

$$
\begin{aligned}
\partial_{t} e+u_{j} \partial_{j} e & =\partial_{i} \Psi_{i}-2 \mu S_{i j} S_{i j}-\alpha u_{i} \partial_{j} \tau_{i j}+u_{i} f_{i}, \\
\partial_{t} h+u_{j} \partial_{j} h & =\kappa \phi \partial_{k k} \phi+\alpha u_{i} \partial_{j} \tau_{i j},
\end{aligned}
$$

where $\Psi_{i}=u_{i}\left(-p^{\prime}+h\right)+2 \mu u_{j} S_{i j}$, and $-p^{\prime}+h=-p+\alpha\left(\partial_{i} c\right)^{2}$. The free energy equation has been transformed into an advection equation, where the first term in the right-hand side represents the diffusive and dissipative action of the chemical potential, and the 
second term the interaction of the fluid-fluid interface with the velocity field through the stress tensor, $\tau_{i j}$. This term also appears in the kinetic energy equation, and represents the conservative energetic exchange between the free energy of the interface and the kinetic energy of the flow.

By taking the volume average of 2.10 and 2.11 , we obtain an equation for the evolution of the total kinetic energy and the surface energy, $\mathcal{E}=\langle e\rangle_{V}$ and $\mathcal{H}=\langle h\rangle_{V}$,

$$
\begin{aligned}
d_{t} \mathcal{H} & =\kappa\left\langle\phi \partial_{k k} \phi\right\rangle_{V}-\left\langle\alpha u_{i} \partial_{j} \tau_{i j}\right\rangle_{V}, \\
d_{t} \mathcal{E} & =2\left\langle\mu S_{i j} S_{i j}\right\rangle_{V}+\left\langle\alpha u_{i} \partial_{j} \tau_{i j}\right\rangle_{V}+\left\langle u_{i} f_{i}\right\rangle_{V},
\end{aligned}
$$

where the spatial fluxes vanish after averaging provided that there are no fluxes through the boundaries. This is the case for the periodic boundary conditions. Note that by virtue of (2.8), the total surface energy is $\mathcal{H}=\sigma A$, where $A$ is the surface of the fluid-fluid interface. On average, $\alpha u_{i} \partial_{j} \tau_{i j}$ is the only term responsible for the exchange between the surface energy and kinetic energy.

\subsection{The physical mechanism of the surface energy variations}

To gain a better physical understanding of the energy-exchange term, we further expand it into

$$
\alpha u_{i} \partial_{j} \tau_{i j}=\alpha \partial_{j}\left(u_{i} \tau_{i j}\right)-\alpha S_{i j} \tau_{i j}
$$

The first term in the right-hand side represents the divergence of a flux and vanishes in the mean, so that only the second term in the right-hand side has a net non-zero contribution. Furthermore, due to the symmetric form of $\tau_{i j}$, only the symmetric part of the velocity gradient tensor, the rate-of-strain tensor $S_{i j}$, interacts with $\tau_{i j}$. Considering that the components of the vector normal to the surface tangent plane are $n_{i}=\partial_{i} c / \gamma$, where $\gamma=\sqrt{\left(\partial_{k} c\right)^{2}}$, we rewrite the Korteweg stress tensor as $\tau_{i j}=-\alpha \gamma^{2} n_{i} n_{j}$, and the exchange term as $-\alpha \gamma^{2} n_{i} S_{i j} n_{j}$. This term describes the change in the free energy per unit volume. We transform it into a change of energy per unit area by integrating it normal to the interface, which yields

$$
\vartheta=-\sigma n_{i} S_{i j} n_{j}
$$

Here we have assumed that the interface is in equilibrium, so that 2.4 holds, and that neither $\boldsymbol{n}$ nor $S_{i j}$ change substantially across the interface width. Both assumptions are fulfilled in the sharp-interface limit (Magaletti et al.|2013).

Introducing the above analysis in the evolution equations for the kinetic energy and the surface energy, we obtain

$$
\begin{aligned}
d_{t} \mathcal{H} & =\kappa\left\langle\phi \partial_{k k} \phi\right\rangle_{V}+\langle\vartheta\rangle_{S}, \\
d_{t} \mathcal{E} & =2\left\langle\mu S_{i j} S_{i j}\right\rangle_{V}-\langle\vartheta\rangle_{S}+\left\langle u_{i} f_{i}\right\rangle_{V},
\end{aligned}
$$

where $\langle\cdot\rangle_{S}$ denotes the integral over the fluid-fluid surface. On average, $\vartheta$ is the only term responsible for the exchange of surface and kinetic energies. These equations are valid independently of the physical properties of the fluids in the mixture. In the case of different viscosities, the balance of tangential stresses produces a discontinuity in the rateof-strain tensor across the interface (in the sharp-interface limit), but this discontinuity only affects the off-diagonal components of the rate-of-strain tensor in a frame fixed to the interface normal vector, which do not enter $\vartheta$ (Dopazo et al. 2000$)$. Thus the expression for $\vartheta$ remains valid and has equal value at both sides of the interface.

The expression in 2.15 resembles the vortex stretching term in the evolution equation of the enstrophy (the square of the vorticity vector), which is responsible for its amplification (Betchov 1956). By analogy, $\vartheta$ describes the stretching or contraction of the 
(a)

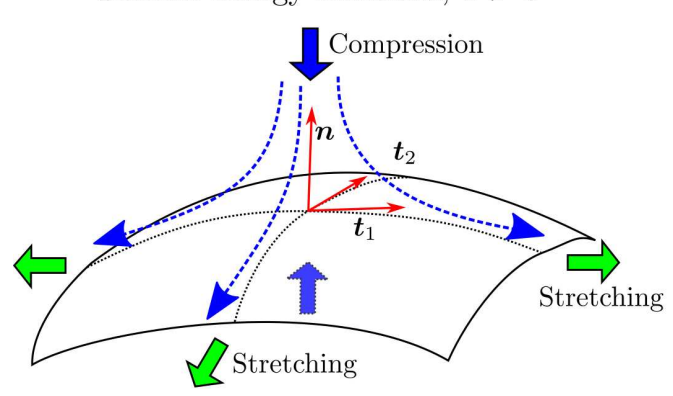

(b)

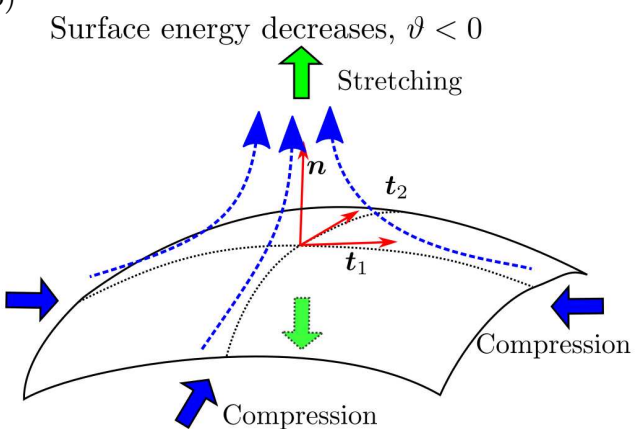

Figure 1. (a,b) Schematic representation of the mechanism that generates (a) positive and (b) negative increments of the surface energy due to the compression or stretching of the interface between two inmiscible liquids, where $\boldsymbol{n}$ is the orthogonal vector normal to the interface, $\boldsymbol{t}^{1}$ and $\boldsymbol{t}^{2}$ are vectors parallel to the surface, and $\vartheta=-\sigma n_{i} S_{i j} n_{j}$. Blue and green arrows indicate the compressive and stretching directions of the rate-of-strain tensor at the surface, and dotted lines are the streamlines of the velocity field with respect to the surface.

interface width by the rate-of-strain tensor. This result may be difficult to interpret from a physical and geometrical perspective, because the interface between inmiscible fluids has a width of molecular scale, and its relaxation time is much faster than the time-scale of the velocity gradients. In what follows, we give this term a physical interpretation.

For simplicity and consistency with the numerical simulations shown later in this paper, we have considered incompressible fluids. Nevertheless, 2.15 holds regardless of the divergence of the velocity field. Compressibility modifies both (2.12) and (2.13), since new fluxes and variables appear, but it does not modify the structure of the exchange term, which remains the only net source of energy exchange. To derive 2.15 from $\alpha u_{i} \partial_{j} \tau_{i j}$, we have only assumed that the gradients of the velocity field take place at a scale much larger than the interface thickness. Thus, 2.15 is also valid for compressible flows as long as the velocity field does not contain any discontinuity (shock) in the interface. For the sake of generality we consider, in the following analysis, a compressible flow, and decompose the rate-of-strain tensor into a deviatoric and a volumetric part, $S_{i j}=S_{i j}^{d}+(P / 3) I_{i j}$, where $I_{i j}$ is the identity tensor, and $P=S_{l k} I_{l k}$ is the local volumetric rate of expansion or contraction. Since $S_{i j}^{d} I_{i j}=0$ and $I_{i j} n_{j} n_{i}=1$, we arrive at

$$
S_{i j} n_{j} n_{i}=S_{i j}^{d}\left(n_{j} n_{i}-I_{i j}\right)+P / 3 .
$$

Now we reformulate 2.15 in terms of $P$ and of any pair of orthonormal vectors parallel to the surface, $\boldsymbol{t}^{1}$ and $\boldsymbol{t}^{2}$

$$
\vartheta=\sigma\left(t_{k}^{1} S_{k j}^{d} t_{j}^{1}+t_{k}^{2} S_{k j}^{d} t_{j}^{2}\right)-\sigma P / 3
$$

where we have considered that, $n_{j} n_{i}-I_{i j}=-t_{i}^{1} t_{j}^{1}-t_{i}^{2} t_{j}^{2}$. The first term represents the growth rate of an infinitesimal surface area, $\delta A$, due to the deviatoric part of the rate-of-strain tensor, which reads

$$
\frac{1}{\delta A} \mathrm{~d}_{t} \delta A=t_{k}^{1} S_{k j}^{d} t_{j}^{1}+t_{k}^{2} S_{k j}^{d} t_{j}^{2}
$$

The second term is related to compressibility effects, and is proportional to the growth rate of an infinitesimal volume at the surface.

In the case of an incompressible flow, in which $P=0$ and $S_{i j}=S_{i j}^{d}$,

$$
\vartheta=\sigma\left(t_{k}^{1} S_{k j} t_{j}^{1}+t_{k}^{2} S_{k j} t_{j}^{2}\right)
$$


which shows that the surface energy increases as a consequence of the stretching of the surface area by the rate-of-strain tensor. The opposite mechanism is also possible, and the decrements of the surface energy are related to the contraction of the surface area. In figure $1(\mathrm{a}, \mathrm{b})$ we show a schematic representation of these mechanisms for an incompressible flow.

\section{Single-drop experiments in homogeneous isotropic turbulence}

The analysis presented in the previous section is valid for any configuration of the fluid-fluid interface, or flow regime. Hereinafter we apply our analysis to the dynamics of a single drop embedded in a homogeneous and isotropic turbulent flow.

\subsection{Numerical method}

We consider two fluids with equal density and kinematic viscosity, and integrate (2.1) in a triply periodic cubic domain of volume $L^{3}=(2 \pi)^{3}$ by projecting the equations on a basis of $N / 2$ Fourier modes in each direction, where $N=256$. Non-linear terms are computed through a dealiased pseudo-spectral procedure, and a third-order semi-implicit Runge-Kutta scheme is used for the time integration, with a decomposition of the linear terms proposed by Badalassi et al. (2003). To sustain turbulence in a statistically steady state, we implement a linear body-force, $\widehat{f}_{i}=C_{f} \widehat{u}_{i}$, that is only applied to wavenumbers $k<2$, where $\hat{\imath}$ denotes the Fourier transform and $k$ is the wavenumber magnitude. The forcing coefficient, $C_{f}$, is set so that, at each time, the total kinetic energy per unit time injected in the system is equal to a constant, which is chosen so as to fix the numerical resolution, $k_{\max } \eta=4$, where $\eta=\left(\nu / \varepsilon^{4}\right)^{3 / 4}$ is the Kolmogorov length scale and $k_{\max }=N / 3$ is the maximum wavenumber magnitude after dealiasing.

The Reynolds number of the flow is $R e_{\lambda}=\lambda u^{\prime} / \nu=58$, where $\lambda=\sqrt{15(\nu / \varepsilon)} u^{\prime}$ is the Taylor microscale, $u^{\prime}=\sqrt{2 \mathcal{E} / 3}$ is the root-mean-square of the velocity fluctuations, and $\mathcal{E}=1 / 2\left\langle u_{i} u_{i}\right\rangle$ is the ensemble-averaged kinetic energy. The thickness of the fluid-fluid interface is set by the Cahn number $\mathrm{Ch}=\left(\alpha / \beta L^{2}\right)^{1 / 2}=0.012$, which for $N=256$ is appropriate to resolve the interface with a spectral Fourier basis (Chen \& Shen 1998). The mobility $\kappa$ defines the Peclet number, $\mathrm{Pe}=u^{\prime} L^{2} / \kappa \sqrt{\alpha \beta}=3 \mathrm{Ch}^{-2}$, which is fixed to ensure that the dynamics of the interface are consistent in the sharp-interface limit (Magaletti et al. 2013). The time-step is set to $\Delta t=0.04 \mathrm{Ch}$. Simulations have been performed on GPUs using a modified version of the spectral code described in Cardesa et al. (2017).

The code has been validated against Shao et al. (2018), and the results of this validation analysis are presented in appendix I. In addition, the consistency of the numerical parameters, such as the time-step and the spatial resolution, have been checked.

\subsection{Initial conditions and drop size}

We introduce a drop of diameter $d=\frac{1}{3} L=45 \eta$ in a fully developed turbulent flow at time $t=0$, and integrate the governing equations (2.1) until the drop breaks at time $t_{b}$. In figure 2, we show a visualisation of a typical single-drop simulation. Since $d \gg \eta$, breakup is dominated by inertial forces and characterised by the Weber number, $W e=\rho \varepsilon^{2 / 3} d^{5 / 3} / \sigma$, and by a characteristic inertial time scale

$$
t_{d}=\left(d^{2} \varepsilon\right)^{1 / 3} .
$$

To statistically characterise drop deformation, we perform many independent single-drop simulations initialised with statistically independent turbulent flow fields. Mass leakage 

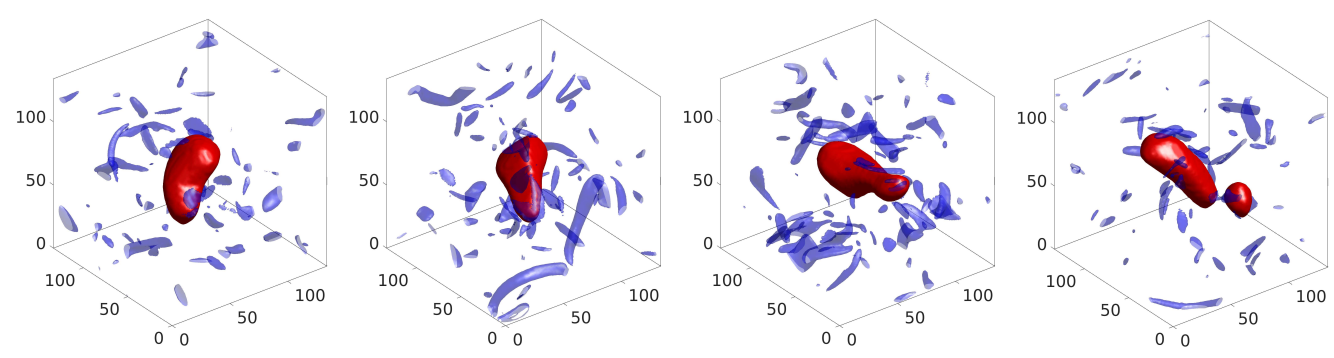

Figure 2. Temporal evolution of a drop at $W e=1.8$. The frame is fixed at the centre of the drop, and the time of the snapshots corresponds, from left to right, to $t / t_{d}=2.0,2.9,4.8$, and 5.1. Blue isosurfaces denote vorticity with magnitude $|\boldsymbol{\omega}|=2.6\langle|\boldsymbol{\omega}|\rangle$, where the brackets denote the ensemble average. The size of the computational box is marked in Kolmogorov units.

(a)

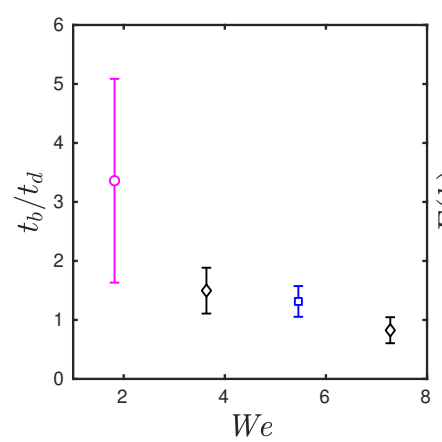

(b)

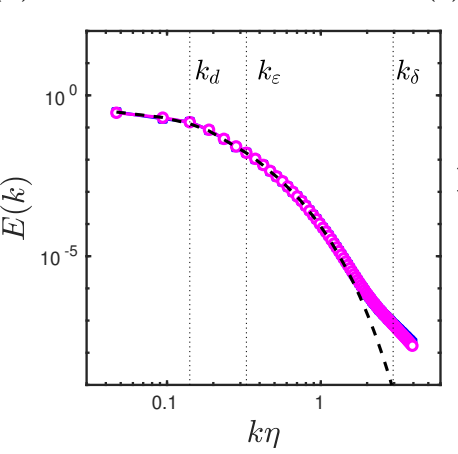

(c)

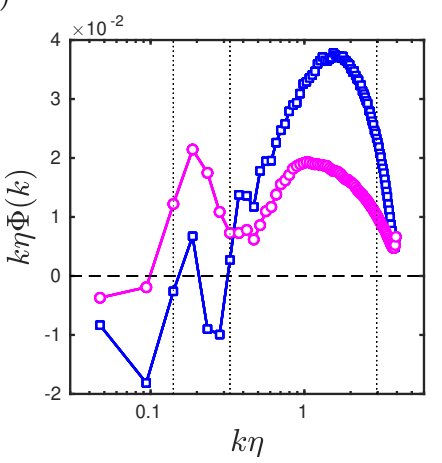

Figure 3. (a) Median time to breakup as a function of the We. The upper and lower bars mark plus-minus the standard deviation. The style of the markers is only used for ease of reference for the data shown in the following panels. (b) Average kinetic energy spectra at $R e_{\lambda}=58$ for: --- , turbulent flow without droplet; $-0-W e=1.8 ;-\square-, W e=5.4$. Vertical dotted lines mark the scale (wavenumber) of the drop, $k_{d}=2 \pi / d$, the scale where the spectral density of the kinetic energy dissipation is the highest, $k_{\varepsilon}$, and the scale of the interface, $k_{\delta}=2 \pi / \delta$. The spectra are averaged across the flow fields in the database. (c) Pre-multiplied production spectra, $k \eta \Phi(k)$. Lines as in (b). The total contribution of each wavenumber to the variation of the surface free energy is represented as the area below the curve. $\Phi$ is normalised with its average in each case.

(Yue et al. 2007) leads to a slow but progressive reduction of the drop diameter, and to a time-dependent Weber number, $W e^{\dagger}(t)$, which decreases slightly through the simulations. However, this variation is small, and therefore we do not employ any special numerical approach to prevent it (Zhang \& Ye 2017; Soligo et al. 2019). We consider the effective Weber number of our simulations as the average Weber number at the average time of breakup, $\left\langle t_{b}\right\rangle$, i.e $W e=\left\langle W e^{\dagger}\left(\left\langle t_{b}\right\rangle\right)\right\rangle$. Here the brackets denote the ensemble average. The difference between the time-dependent Weber number at the time of breakup, $W e^{\dagger}\left(t_{b}\right)$, and the effective Weber number, $W e$ is at most $\sim 3 \%$ in the worst cases.

We have performed simulations at four different effective $W e \in[1.8,7.4]$. For each $W e$, the number of simulations is approximately 100, which yields a total simulation time of between $300 t_{d}$ and $1500 t_{d}$.

From each simulation, we stored a sufficient number of full flow fields to have fully converged statistics. We analysed flow fields at times at least $0.5 t_{d}$ after the initial condition and before breakup. We assume that in this time interval the evolution of 
the drop is statistically stationary. In this range of $W e$, breakup occurs in times of the order of the inertial time-scale of the drop, $t_{d}$. In figure 3 (a), we show the median and the standard deviation of the time to breakup, $t_{b}$, as a function of the $W e$. As expected, lower values of the $W e$ yield longer times to breakup.

In what follows we show that although $d$ is comparable to the integral scale of the flow, the drop does neither modify the structure of the surrounding turbulence nor resonates with the numerical box. The drop interacts mostly with scales smaller than $d$, indicating that the linear forcing used to sustain turbulence does not affect breakup. In figure $3(\mathrm{~b})$, we show the average kinetic energy spectrum, $E(k)=2 \pi k^{2}\left\langle\widehat{u} \widehat{u}^{*}\right\rangle_{k}$, with and without an immersed drop. Here $\langle\cdot\rangle_{k}$ denotes averaging over modes with wavenumber magnitude $k$, and ${ }^{*}$ represents the complex conjugate. The energy spectra are similar for the flow with and without droplet above $k \eta \sim 1$, suggesting a similar turbulent structure in those scales. To further corroborate this, we calculate the skewness and flatness factor of the longitudinal velocity derivatives,

$$
\mathcal{F}_{n}=\frac{\left\langle\left(\partial_{i} u_{i}\right)^{n}\right\rangle}{\left\langle\left(\partial_{i} u_{i}\right)^{2}\right\rangle^{n / 2}},
$$

where no summation is intended for repeated indices. We find that, away from the drop surface, $\mathcal{F}_{3} \approx-0.52$ and $\mathcal{F}_{4} \approx 5.2$ independently of the Weber number, which are similar to the values for a simulation without the drop and in the expected range for the $R e_{\lambda}$ considered (Jiménez et al. 1993).

The good collapse of the energy spectra in the small wavenumbers (large scales) also suggests the absence of any resonances between the drop and the box, which could lead to spurious large-scale dynamics. To examine how the large-scale forcing affects the dynamics of breakup, we study the pre-multiplied production spectra, $k \eta \Phi(k)$, shown in figure $3(\mathrm{c})$, where

$$
\Phi(k)=-4 \pi k^{2} \Re\left\langle\left(\widehat{\left(u_{j} \partial_{j} c\right.}\right) \hat{\phi}^{*}\right\rangle_{k},
$$

describes the contribution of each scale to the changes of the surface energy due to the deformation of the interface by the velocity field. Note that the integral of $\Phi(k)$ across wavenumbers is equal to $\langle\vartheta\rangle_{S}$. Variations of the surface energy are due mostly to turbulent fluctuations at scales below the drop diameter, whereas the contribution of larger scales is comparatively small. In fact, the contribution of the scales affected by the forcing (with $k<2$ ) to the production of surface energy is slightly negative on average, confirming that the forcing does not contribute to deformation and breakup.

\section{Analysis of the energetic exchange in isotropic turbulence}

\subsection{Local and non-local surface stretching}

Despite the simplicity of the energetic exchange described in $\$ 2$, the coupling between the dynamics of the drop surface and the surrounding turbulence is bidirectional and highly non-linear. The rate-of-strain tensor generates surface contraction or expansion, and, at the same time, the surface dynamics generates straining motions. For instance, as a deformed drop relaxes toward a spherical shape, the surface energy follows damped oscillations before settling into equilibrium (see appendix I). Although these changes of the surface energy are produced by the stretching and compression of the surface, they are drop-induced and not necessarily related to drop breakup.

To separate the dynamics of the interface from the action of the surrounding turbulence, we split the surface-stretching term into contributions related to eddies close to (including those inside) the drop, and far from the drop. Here we consider eddies 
(a)

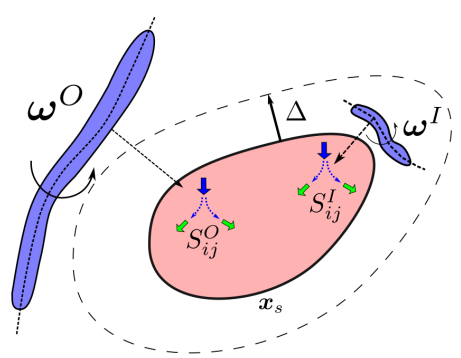

Alberto Vela-Martín and Marc Avila

(b)

(c)

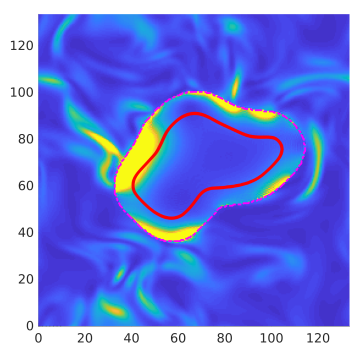

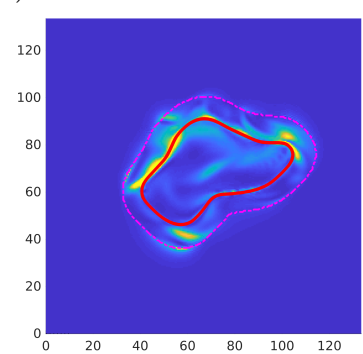

FiguRE 4. (a) Decomposition of the rate-of-strain tensor in contributions due to eddies a distance $\Delta$ from the drop surface, denoted as $S_{i j}^{O}$, and a distance $\Delta$ close to the surface (including the inside of the drop), denoted as $S_{i j}^{I}$. $\boldsymbol{x}_{s}$ marks the drop surface. (b,c) Magnitude of the (b) outer and (c) inner rate-of-strain tensor, $2 S_{i j}^{O} S_{i j}^{O}$ and $2 S_{i j}^{I} S_{i j}^{I}$, in a direct numerical simulation for $\Delta=0.19 d$ and $W e=5.4$. The solid red line marks the drop surface, and the dashed magenta line a distance $\Delta=0.19 d$ from the surface. In (b), the outer rate-of-strain tensor at a distance smaller than $\Delta=0.19 d$ from the drop surface has been multiplied by 10 to ease visualisation. Axes in Kolmogorov units.

as patches of swirling fluid, and associate them with the vorticity field. The kinematic relations that hold between the vorticity vector and the rate-of-strain tensor imply that eddies away from the drop stretch its surface by non-local effects (Ohkitani \& Kishiba 1995. Hamlington et al. 2008). Let us consider a distance $\Delta$, and separate the vorticity field in $\Delta$-outer and $\Delta$-inner eddies. The former are defined as eddies a distance $\Delta$ or further from the drop surface, while the later correspond to eddies that are closer than $\Delta$ or inside the drop surface. We define the vorticity field at a distance further than $\Delta$ from the drop surface as

$$
\boldsymbol{\omega}^{\prime O}=G(\boldsymbol{x} ; \Delta) \boldsymbol{\omega}
$$

where $\boldsymbol{\omega}=\nabla \times \boldsymbol{u}$ is the vorticity vector, and

$$
G(\boldsymbol{x} ; \Delta)=\left\{\begin{array}{l}
1 \text { if }\left|\boldsymbol{x}-\boldsymbol{x}_{s}\right|_{s}>\Delta \text { and } \boldsymbol{x} \in \mathscr{O}, \\
0 \text { if otherwise, }
\end{array}\right.
$$

is a kernel that truncates the vorticity field at a distance $\Delta$ from the drop surface. Here $\boldsymbol{x}_{s}$ defines the surface of the drop, $\left|\boldsymbol{x}-\boldsymbol{x}_{s}\right|_{s}$ is the shortest Euclidean distance from $\boldsymbol{x}$ to the drop surface, and $\mathscr{O}$ comprises all the points outside the drop. Let us note that $\boldsymbol{\omega}^{\prime O}$ does not define a vorticity field because it does not locally fulfil $\nabla \cdot \omega^{O}=0$, where the truncation is performed. We thus project it into the closest divergence-free field, $\boldsymbol{\omega}^{O}=\boldsymbol{\omega}^{\prime O}-\nabla \psi$, by solving

$$
\nabla^{2} \psi=\nabla \cdot \omega^{\prime O}
$$

with periodic boundary conditions. We calculate the stretching induced on the drop by the eddies away from its surface from the Biot-Savart law (Ohkitani \& Kishiba 1995). By taking the curl of the vorticity and considering that $\nabla \cdot \boldsymbol{u}^{\sigma}=0$, we obtain the following equation

$$
\nabla^{2} \boldsymbol{u}^{O}=-\nabla \times \boldsymbol{\omega}^{O}
$$

which, when solved with periodic boundary conditions, provides the rate-of-strain tensor produced by $\Delta$-outer eddies, $S_{i j}^{O}=\frac{1}{2}\left(\partial_{j} u_{i}^{O}+\partial_{i} u_{j}^{O}\right)$, and by $\Delta$-inner eddies, $S_{i j}^{I}=S_{i j}-S_{i j}^{O}$. 
(a)

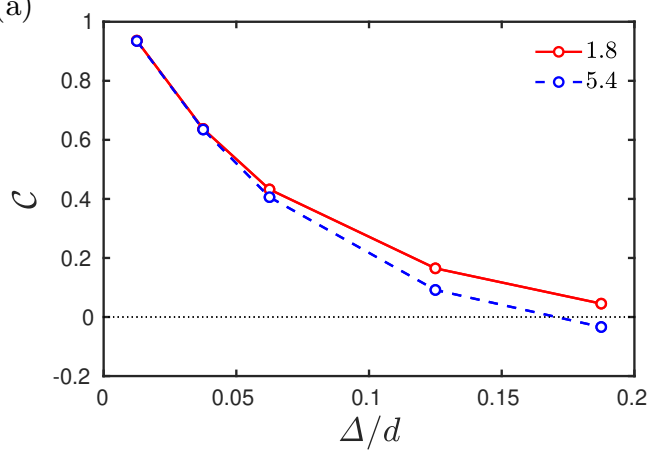

(b)

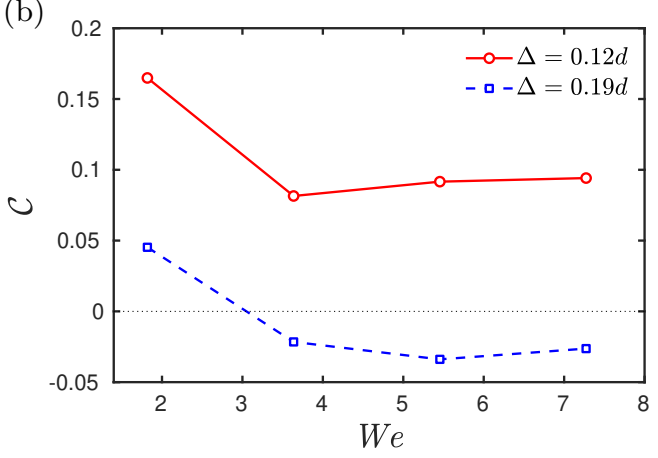

Figure 5. (a,b) Correlation coefficient, $\mathcal{C}$, between the stretching by inner eddies, $\vartheta^{I}$, and outer eddies, $\vartheta^{O}$, as a function of (a) $\Delta$ and (b) We.

The stretching of the drop surface by $\Delta$-outer and $\Delta$-inner eddies are

$$
\begin{aligned}
\vartheta^{O} & =-\sigma n_{i} S_{i j}^{O} n_{j}, \\
\vartheta^{I} & =-\sigma n_{i} S_{i j}^{I} n_{j},
\end{aligned}
$$

respectively, where the rate-of-strain tensor is evaluated at the surface of the drop. A schematic representation of this decomposition and its application to a snapshot of a direct numerical simulation are shown in figures $4(\mathrm{a})-(\mathrm{c})$.

\subsection{Stretching by inner and outer eddies}

In this section, we obtain a value of $\Delta$ for which the separation in $\Delta$-inner and $\Delta$-outer eddies is physically meaningful and practical. We aim to separate the surface stretching into a contribution due to eddies close to the drop surface, which are affected by surface dynamics or by the material properties of the drop, and another contribution due to eddies far from the surface, which are independent of surface dynamics or the material properties of the drop.

A practical approach is to find the smallest $\Delta$ for which the $\Delta$-outer eddies and the surface are not coupled, i.e, the $\Delta$-outer stretching is independent of surface dynamics. In addition, this approach identifies the smallest region close to the drop surface where eddies are affected by surface dynamics. We denote the $\Delta$-inner and $\Delta$-outer eddies identified with this criterion simply as inner and outer eddies.

To find the $\Delta$ that separates inner and outer eddies, we use the correlation coefficient between $\vartheta^{O}$ with $\vartheta^{I}$,

$$
\mathcal{C}=\frac{\left\langle\overline{\vartheta^{I}} \cdot \overline{\vartheta^{O}}\right\rangle}{\left.\sqrt{\left.{\overline{\vartheta^{I}}}^{2}\right\rangle \overline{\vartheta O}^{2}}\right\rangle},
$$

where the bar denotes quantities without their ensemble average, $\bar{\vartheta}=\vartheta-\langle\vartheta\rangle$. We evaluate $\mathcal{C}$ for different $W e$ and $\Delta$, and show the results in figure $5(a, b)$. The correlation coefficient is close to unity for $\Delta$ close to zero, and decays fast with increasing $\Delta$. For $\Delta=0.12 d, \mathcal{C} \approx 0.2$, and for $0.19 d$ it drops close to zero. In figure 5 (b), we show that the correlation coefficient for $\Delta=0.12 d$ and $\Delta=0.19 d$ is close to zero for all $W e$, indicating that $0.12 d<\Delta<0.19 d$ is a reasonable estimate of the distance that separates outer and inner eddies in the range of $W e$ considered here. This separation corresponds in Kolmogorov units to $6 \eta<\Delta<9 \eta$.

In the following, we will study the contribution of inner and outer eddies to the surface 
(a)

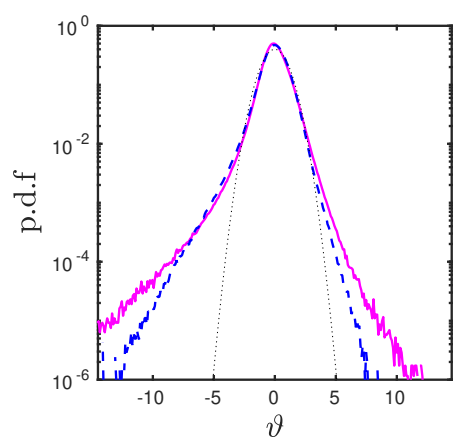

(b)

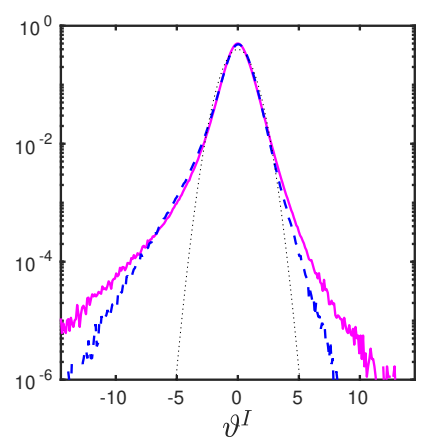

(c)

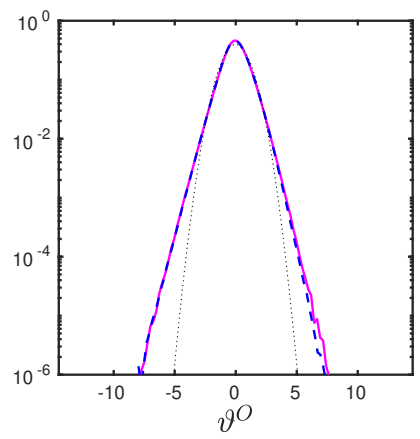

Figure 6. Probability density function of the local surface stretching due to (a) the full flow, $\vartheta$, (b) the inner eddies, $\vartheta^{I}$, and (c) the outer eddies, $\vartheta^{O}$, for $\Delta=0.12 d$ and at $W e=:-$, 1.8; - - - 5.4. Quantities plotted without the mean and divided by their standard deviation.

stretching. For simplicity, we name these contributions as the inner and outer stretching, respectively.

\subsection{Statistics of the surface stretching}

In figure 6(a)-(c), we show the probability density function (p.d.f) of $\vartheta, \vartheta^{O}$, and $\vartheta^{I}$, measured at the surface of the drop for $W e=1.8$ and 5.4, and $\Delta=0.12 d$. Results are similar for $\Delta=0.19 \mathrm{~d}$. We find that $\vartheta$ and $\vartheta^{I}$ are very similar and display fairly fat-tailed distributions which depend on the $W e$. We suggests that these tails are a consequence of surface tension forces produced by the oscillations of the interface. On the other hand, the p.d.f's of $\vartheta^{O}$ are more symmetric, closer to a Gaussian. Their good collapse at two different $W e$ indicates that the outer stretching is not affected by surface dynamics. These results corroborate that the value of $\Delta$ used to decompose the flow in inner and outer eddies is adequate.

In figure 7 (a), we show the mean of $\vartheta, \vartheta^{O}$, and $\vartheta^{I}$ normalised with $\rho u_{d}^{3}$, where $u_{d}=d / t_{d}$, for different values of $W e$. The average of $\vartheta$ increases with $W e$, which is consistent with the statistics of the breakup time shown in figure 3(a). We assume that the breakup of an initially spherical drop takes place when a critical increment of the surface energy, $\Delta \mathcal{H}_{b}$, is reached (Andersson \& Andersson 2006). Because the surface energy can only increase due to the stretching term, its average measured on the surface of the drop is related to the time to breakup by

$$
t_{b} \sim\left\langle\frac{\Delta \mathcal{H}_{b}}{\vartheta d^{2}}\right\rangle,
$$

which has been estimated by averaging equation 2.12 in time, considering that the increment of the surface energy is approximately $\langle\vartheta\rangle d^{2}$, and neglecting the dissipation of surface energy. The increment of the surface energy required for breakup is approximately the difference between the surface energy after breakup, $\mathcal{H}_{b}$, and the surface energy of a spherical drop, $\mathcal{H}_{0}$, i.e., $\Delta \mathcal{H}_{b} \approx \mathcal{H}_{b}-\mathcal{H}_{0}=f \sigma d^{2}$, where $f \leqslant f_{b}$ is a factor that depends on the geometry of breakup and is largest for binary breakup, when $f_{b}=2^{1 / 3}-1 \approx 0.26$ (Andersson \& Andersson 2006). The time to breakup increases with decreasing $W e$, which is consistent with the increment of $\langle\vartheta\rangle$ with $W e$.

The average outer stretching is positive and larger than the inner stretching. It decreases approximately by a factor of 2 from $W e=1.8$ to $W e=7.4$. The inner stretching increases substantially with $W e$, transitioning from $\left\langle\vartheta^{I}\right\rangle\left\langle 0\right.$ to $\left\langle\vartheta^{I}\right\rangle>0$ at approximately $W e \approx 3.5$. Note that negative average values of the inner stretching 
(a)

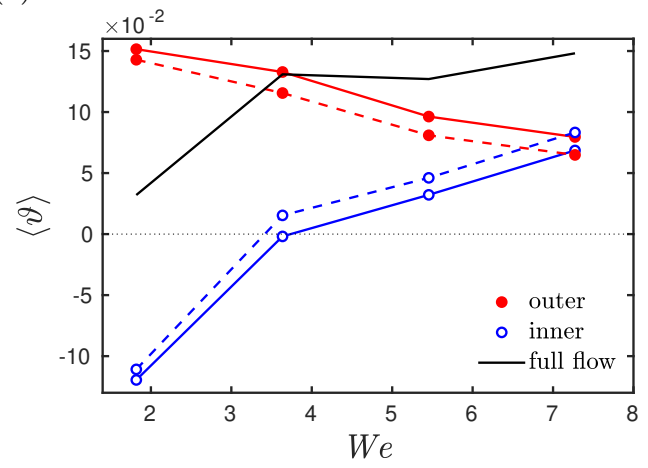

(c)

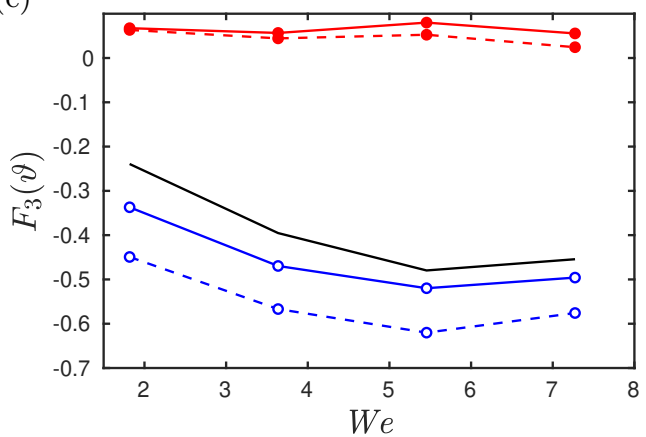

(b)

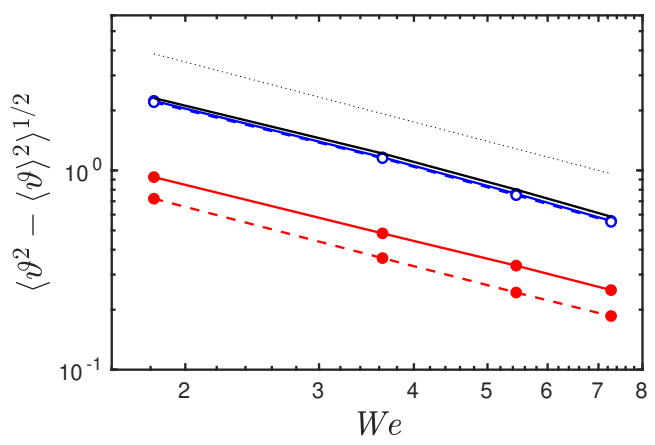

(d)

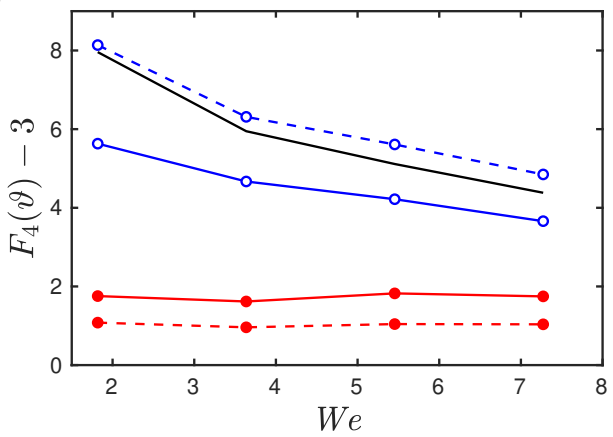

Figure 7. (a) Mean, (b) standard deviation, (c) skewness and (d) excess flatness of $\vartheta$ as a function of $W e$. Mean and standard deviation normalised with $\rho u_{d}^{3}$, where $u_{d}=d / t_{d}$. Solid symbols corresponds to the statistics of $\vartheta^{O}$, and empty symbols to $\vartheta^{I}$. Colour lines correspond to $\Delta=:-, 0.12 d ;---, 0.19 d$; and solid black line to the full rate-of-strain tensor. In (b) the dotted line is proportional to $W e^{-1}$.

indicates that, close to the surface, energy is being mostly transferred from the surface to turbulent fluctuations. This phenomenon will be examined in detail in 4.5 .

In figure $7(\mathrm{~b})$, we show the standard deviation of $\vartheta$ normalised with $\rho u_{d}^{3}$. It is approximately proportional to $W e^{-1}$, and substantially higher for the inner than for the outer stretching. In all cases, the standard deviation of the surface stretching is larger than its mean. This is particularly significant for the inner stretching and small $W e$, and suggests that a very significant part of the stretching is not efficient and cancels out when averaging.

We focus now on higher-order statistics of the surface stretching, in particular the skewness and the flatness factor, which are defined as

$$
F_{n}(\vartheta)=\frac{\left\langle(\vartheta-\langle\vartheta\rangle)^{n}\right\rangle}{\left\langle\vartheta^{2}-\langle\vartheta\rangle^{2}\right\rangle^{n / 2}}
$$

for $n=3$, and $n=4$ respectively, and are shown in figures $7(\mathrm{c}, \mathrm{d})$. For ease of comparison, we consider the excess flatness factor, which is the flatness factor minus that of a Gaussian distribution, for which $F_{4}=3$. These statistical moments reflect the strong differences between inner and outer stretching. While the skewness of the outer contributions is close to zero and does not change with $W e$, the inner stretching is negatively skewed, and its skewness depends on the $W e$. The excess flatness factor conveys a similar picture. The outer contributions have a low, $W e$-independent, excess flatness factor, whereas for the 
(a)

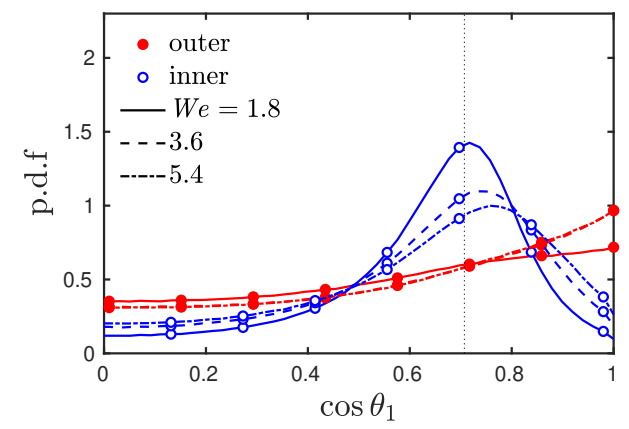

(c)

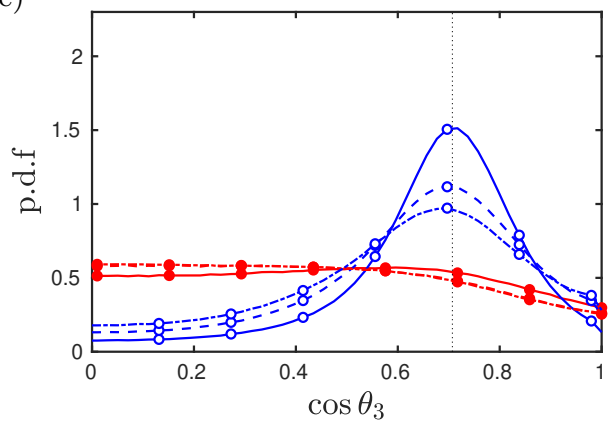

(b)

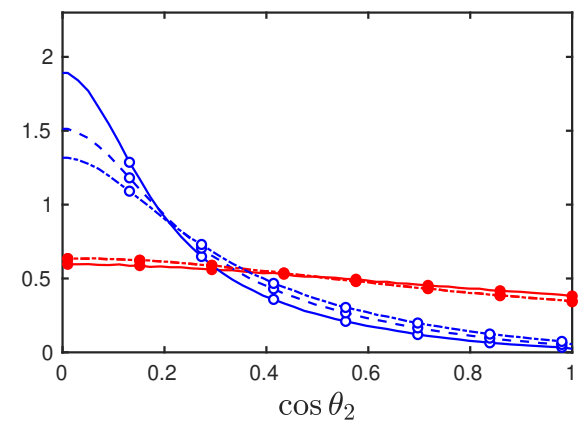

(d)

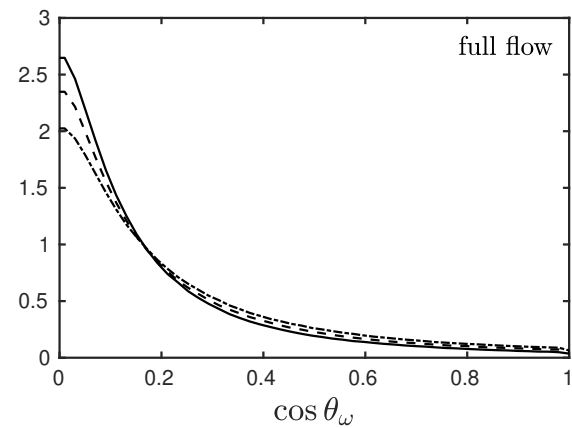

Figure 8. (a)-(c) Probability density function of $\cos \theta_{i}=\boldsymbol{n} \cdot \boldsymbol{v}_{i}$, where $\boldsymbol{v}_{i}$ are the principal directions of the rate of strain tensor and $\lambda_{1} \leqslant \lambda_{2} \leqslant \lambda_{3}$ are their eigenvalues. Solid markers correspond to angles calculated with $S_{i j}^{O}$, and empty markers to $S_{i j}^{I}$, for $\Delta=0.19 d$. (d) Similar but for the vorticity vector, $\cos \theta_{\omega}=\boldsymbol{n} \cdot \boldsymbol{\omega} /|\boldsymbol{\omega}|$. Lines correspond to $W e=:-, 1.8 ;---$, $3.6 ;-\cdots, 5.4$. The vertical dotted line in (a) and (c) marks $\cos \pi / 4$.

inner contributions the excess flatness factor increases with decreasing $W e$. These results corroborate, first, that the statistics of the outer stretching are $W e$-independent, and, second, that the inner stretching has an intermittent structure, with intense events of the surface stretching becoming stronger with decreasing $W e$. These events are probably related to the relaxation of the drop towards a spherical shape.

\subsection{Geometrical characterisation of the inner and outer stretching}

To further explain the differences between the inner and outer stretching, and how they change with $W e$, we study the structure of the vorticity vector and the rate-of-strain tensor induced by inner and outer eddies on the surface of the drop. In the spirit of the analysis of vortex stretching in isotropic turbulence (Ashurst et al. 1987, Buaria et al. 2020), we analyse the surface stretching term in the frame of reference of the eigenvectors of the rate-of-strain tensor, and quantify the contribution of each of its eigenvalues to the total surface stretching.

In figure 8(a)-(c), we show the p.d.f of the cosine of the angle of alignment between each of the principal axes of the rate-of-strain tensor, $\boldsymbol{v}_{1}, \boldsymbol{v}_{2}$ and $\boldsymbol{v}_{3}$ (where $\lambda_{1} \leqslant \lambda_{2} \leqslant \lambda_{3}$ are their corresponding eigenvalues) and the normal to the surface, $\boldsymbol{n}$. For the inner contributions, the most stretching $\left(\boldsymbol{v}_{3}\right)$ and the most compressing $\left(\boldsymbol{v}_{1}\right)$ eigenvectors tend to be oriented at $\sim 45^{\circ}$ with respect to the surface normal, and therefore also to the surface tangent plane, while the intermediate eigenvalue is predominantly normal to the surface normal (parallel to the surface tangent plane). As shown in figure 8(d), also the 
(a)

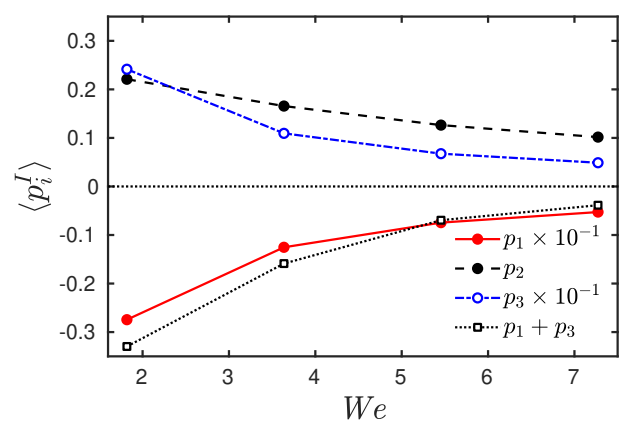

(c)

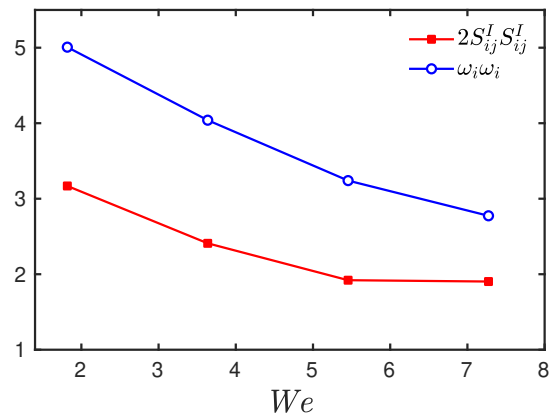

(b)

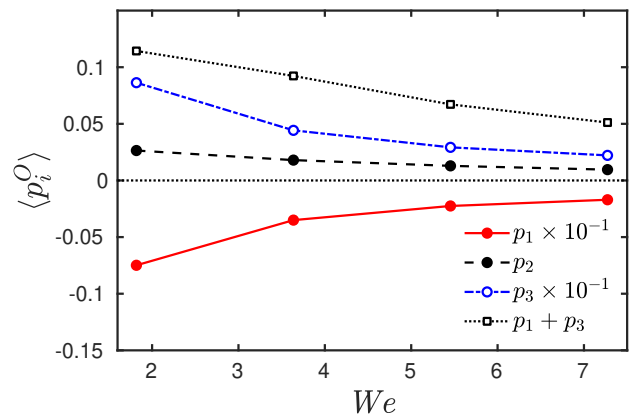

(d)

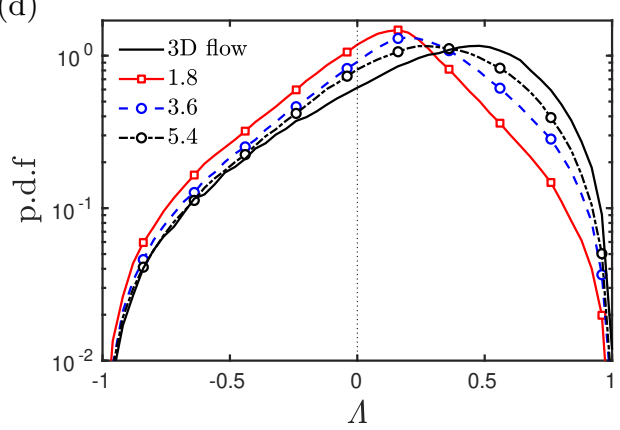

Figure 9. (a,b) Average surface stretching due to each eigenvalue of the rate-of-strain tensor, $p_{i}=\sigma \lambda_{i} \sin ^{2} \theta_{i}$ as a function of the Weber number for the inner (a) and the outer (b) contributions, for $\Delta=0.19 d$. The surface stretching is normalised with $\rho u_{d}^{3}$. (c) Surface average of the square of the inner rate-of-strain tensor, $2 S_{i j}^{I} S_{i j}^{I}$, for $\Delta=0.19 d \eta$, and the vorticity vector $\omega_{i} \omega_{i}$ for different Weber numbers, normalised with Kolmogorov units. (d) p.d.f of $\Lambda=\log _{2}\left|\lambda_{1}\right| / \lambda_{3}$ for different Weber numbers.

vorticity vector aligns strongly normal to $\boldsymbol{n}$, and parallel to the surface. This trend was also reported by Soligo et al. (2020). In all cases, the alignment is more marked for small $W e$, revealing an important effect of surface dynamics on the configuration of the velocity gradients at the surface of the drop.

The stretching of the surface by outer eddies shows a substantially different picture. There is a weak but consistent tendency of the most compressing eigenvector to align normal to the surface tangent plane. The collapse of the p.d.f's of $\cos \theta_{i}$ at different $W e$ suggests that this effect is independent of the surface dynamics.

We further decompose the surface stretching term into the contribution of each of its eigenvalues. A possible approach is to consider the rate of compression or stretching of the vector normal to the surface by each eigenvalue, $r_{i}=-\sigma \lambda_{i} \sin \theta_{i}$ (no summation for repeated indices is intended), which yields

$$
\vartheta=r_{1}+r_{2}+r_{3}=-\sigma\left(\lambda_{1} \cos ^{2} \theta_{1}+\lambda_{2} \cos ^{2} \theta_{2}+\lambda_{3} \cos ^{2} \theta_{3}\right) .
$$

However this decomposition cannot be readily interpreted from a physical perspective. Instead, we consider the contribution of each eigenvalue to the stretching or compression of the surface tangent plane, $p_{i}=\sigma \lambda_{i} \sin ^{2} \theta_{i}$. Note that $\sin \theta_{i}=\cos \left(\pi / 2-\theta_{i}\right)$ is the projection of each eigenvector on the surface tangent plane. Since $\lambda_{1}+\lambda_{2}+\lambda_{3}=0$, and 
$\cos ^{2} \theta_{i}=1-\sin ^{2} \theta_{i}$, the total surface stretching reads,

$$
\vartheta=p_{1}+p_{2}+p_{3}=\sigma\left(\lambda_{1} \sin ^{2} \theta_{1}+\lambda_{2} \sin ^{2} \theta_{2}+\lambda_{3} \sin ^{2} \theta_{3}\right) .
$$

In figures $9(\mathrm{a}, \mathrm{b})$, we show the averages of $p_{i}$ for the inner and outer contributions. In both cases the surface stretching and compression due to $p_{1}$ and $p_{3}$ is much larger in absolute value than that of $p_{2}$ (note that in the plot $p_{1}$ and $p_{3}$ are divided by 10 for ease of visualisation). However, there is a significant cancellation between $p_{1}$ and $p_{3}$, and $\left\langle p_{1}+p_{3}\right\rangle \sim\left\langle p_{2}\right\rangle$.

For the outer stretching, $p_{1}+p_{3}$ produce net surface stretching, which stems from the tendency of $\boldsymbol{v}_{3}$ to align parallel to the surface (or $\boldsymbol{v}_{1}$ normal to it). Conversely, $p_{2}$ does not contribute on average to the outer stretching. This is in agreement with the almost random orientation of $\boldsymbol{v}_{2}$ with respect to the surface normal, as shown in figure $8(\mathrm{~b})$.

For the inner stretching, the cancellation of $p_{1}$ and $p_{3}$ is consistent with a predominant alignment of $\boldsymbol{v}_{1}$ and $\boldsymbol{v}_{3}$ at $\sim 45^{\circ}$ with respect to the normal and to the surface tangent plane. In this case, $p_{2}$ contributes to the average stretching of the surface. As $W e$ increases, the absolute value of $\left\langle p_{1}+p_{3}\right\rangle$ decreases, and $\left\langle p_{2}\right\rangle$ progressively dominates the average stretching, leading to $\left\langle\vartheta^{I}\right\rangle>0$ for $W e>3.5$, as shown in figure 7 (a).

\subsection{Transition from a $2 D$ to a $3 D$ structure the inner rate-of-strain tensor}

In this section, we explain the transition from $\left\langle\vartheta^{I}\right\rangle\left\langle 0\right.$ to $\left\langle\vartheta^{I}\right\rangle>0$ at $W e \approx 3.5$ by analysing the intensity and structure of the velocity gradients at the drop surface. In figure 9 (c), we show the average of $2 S_{i j}^{I} S_{i j}^{I}$ for $\Delta=0.19 d$, and $\omega_{i} \omega_{i}$, measured at the surface of the drop. The intensity of the velocity gradients on the surface of the drop increase with decreasing $W e$ due to surface tension forces, but these forces impose a strong constraint on their structure. This is particularly noticeable in the rate-of-strain tensor, which has a quasi-2D structure at the low $W e$, with $\left|\lambda_{2}\right| \ll\left|\lambda_{3}\right| \approx\left|\lambda_{1}\right|$, and becomes progressively 3D, $\left|\lambda_{2}\right| \sim\left|\lambda_{3}\right| \sim\left|\lambda_{1}\right|$, as $W e$ increases. To characterise this transition we analyse

$$
\Lambda=\log _{2} \frac{\left|\lambda_{1}\right|}{\lambda_{3}}
$$

at the drop surface. Since $\frac{1}{2} \lambda_{3} \leqslant\left|\lambda_{1}\right| \leqslant 2 \lambda_{3}$, this quantity is defined in the range $-1 \leqslant \Lambda \leqslant 1$. Predominant $\Lambda>0$ is characteristic of fully developed 3D turbulence, and implies that $\lambda_{2}>0$ on average. Conversely, $\Lambda=0$ indicates $2 \mathrm{D}$ dynamics, in which $\lambda_{1}=-\lambda_{3}$ and, by continuity, $\lambda_{2}=-\lambda_{1}-\lambda_{3}=0$. As shown in figure $9(\mathrm{~d})$, the p.d.f of $\Lambda$ shows that, for small $W e,\left|\lambda_{1}\right| \sim \lambda_{3}$ and $\lambda_{2} \ll \lambda_{3}$. As $W e$ increases, the peak of the p.d.f of $\Lambda$ moves towards $\Lambda \approx 0.4$, indicating a transition from a quasi-2D to a $3 \mathrm{D}$ structure of the inner rate-of-strain tensor at the surface. This transition implies that $\lambda_{3}$ and $\left|\lambda_{1}\right|$ decrease with respect to $\lambda_{2}$, which leads to $\left\langle p_{2}\right\rangle>\left\langle p_{1}+p_{3}\right\rangle$, and to $\left\langle\vartheta^{I}\right\rangle>0$.

\section{Discussion}

We have separated the stretching of the drop surface in contributions from inner and outer eddies, which we have defined in 84.2 . For distances to the drop surface larger than $\Delta=0.12 d$, the inner and outer stretching are not correlated pointwise (figure 5 , indicating that beyond this distance the outer stretching is independent of surface dynamics. This is corroborated by the statistics of the outer surface stretching for this $\Delta$, which are roughly $W e$-independent. We have shown that the outer stretching is dominant and contributes on average to the increment of the surface energy at all $W e$.

Conversely, the average of the inner stretching is negative for $W e<3.5$, indicating a 
predominant flux of energy from the surface into turbulent fluctuations. We interpret this flux of energy as the generation of inner eddies due to the relaxation of the surface. Above $W e \approx 3.5$, the average inner stretching becomes positive, indicating that, on average, also inner eddies contribute to drop deformation. In all cases, the inner stretching shows nonGaussian and negatively-skewed We-dependent statistics. Negative events of the inner stretching become more intense as $W e$ decreases, evidencing the action of the surface to restore deformations by transferring the surface energy back to turbulent fluctuations.

At low $W e$, the net increments of the total surface energy depend on the interplay between the outer surface stretching, which constitutes a mechanism of net drop deformation, and the ability of the surface to restore this deformation by transferring energy to inner eddies, which provide a source of energy dissipation through molecular viscosity. In addition, we have shown that at low $W e$ the rate-of-strain tensor is constrained by surface tension forces. Although its average magnitude is larger than in the turbulent background, providing enhanced dissipation of turbulent kinetic energy, its 2D structure and its configuration with respect to the interface precludes the net stretching of the surface. As $W e$ increases, the inner rate-of-strain tensor develops a 3D structure in which its intermediate eigenvalue becomes predominantly positive, producing net stretching of the surface. This phenomenon explains the transition at $W e \approx 3.5$ from a net energy transfer from the surface to inner eddies (negative inner stretching), to a net energy transfer from inner eddies to the surface (positive inner stretching).

We have reported a statistically significant alignment of the surface normal vector with the most compressing direction of the rate-of-strain tensor induced by outer eddies, and of the surface tangent plane with the most stretching one. This phenomenon, which is also observed in the evolution of passive material surfaces in turbulence, and naturally leads to these alignments (Girimaji \& Pope 1990), indicates the persistent stretching of the drop surface by outer eddies. The alignment statistics are similar at different $W e$, corroborating that this mechanism is independent of surface dynamics.

\section{Conclusions}

In this work we have analysed the energetics of fluid-fluid interfaces. One of our main contributions is to provide a mathematical description of the mechanism responsible for the exchange between the kinetic energy of the flow and the surface energy of the fluid-fluid interface. We have shown that this exchange occurs due to the stretching or compression of the surface area by the rate-of-strain tensor (see (2.15)-(2.17)), in a mechanism analogous to the stretching of the vorticity field in turbulence. This analytical result highlights the relevant role of straining motions in drop deformation and breakup, in agreement with experiments and theoretical analyses of laminar flows in the Stokes limit (Rallison 1984).

In the context of drop deformation and breakup, this mathematical analysis provides a solid framework to quantify the energetic exchanges between the surface and the kinetic energy fluctuations of the surrounding turbulence. By leveraging the non-local nature of turbulence, we have separated the total stretching into contributions due to inner and outer eddies. We use here the term outer to refer to eddies sufficiently far from the drop surface for their dynamics to be unaffected by surface tension forces. Conversely, we define inner eddies as those close to the surface or inside the drop, whose dynamics are affected either by surface tension forces or by the material properties of the fluid inside the drop. We have shown that the stretching of the drop surface by outer eddies contributes substantially to the deformation of the drop, and is relevant to drop breakup. 
This mechanism constitutes a physically well-defined, quantitative reinterpretation of the phenomenological 'collision' of eddies (Luo \& Svendsen 1996).

Our results justify a 'stochastic' approach to breakup modelling, particularly at low $W e$. Outer eddies provide the main source of drop deformation. They are not locally coupled to inner eddies or to the dynamics of the interface, which precludes synergies between inner and outer eddies that may lead to enhanced breakup (at least in the case of similar density and viscosity investigated here). For sufficiently long breakup times, the stretching by outer eddies may be modelled as a stochastic forcing with statistical properties that depend mostly on the surrounding turbulence. Although the surface stretching by inner and outer eddies is not coupled locally, it is likely globally coupled, for instance, due to energy conservation.

Our results suggest that for low $W e(W e<3.5)$ drop deformation and breakup occurs as an interplay between inner and outer dynamics, where the latter is the driver and a source of surface energy increments, while the former is a sink. The stretching by outer eddies is mostly balanced by an energy transfer from the surface to inner eddies, which provide a source of dissipation and prevent the buildup of surface energy.

Although our study is limited to the ideal case of incompressible fluids with equal density and viscosity, the theoretical analysis in $\$ 4$ applies equally to compressible fluids, and to fluid pairs with different viscosity and density. For instance, we suggest that an increment of the drop viscosity affects the inner eddies due, first, to the kinematic relations imposed on the rate-of-strain tensor across the interface, which depend on the viscosity ratio (Dopazo et al. 2000), and second, by the enhanced ability of the fluid inside the drop to diffuse momentum and dissipate energy. These changes may inhibit the stretching of the surface by inner eddies and/or provide a fast mechanism to dissipate the surface energy produced by the stretching of outer eddies, explaining the resistance of viscous drops to breakup (Calabrese et al. 1986; Roccon et al. 2017). Our analysis also applies to bubbles. We suggest that our decomposition would allows to separate the effect of the bubble's natural oscillations from that of turbulence, allowing to explore how resonances with the characteristic oscillatory frequency affect breakup (Risso \& Fabre 1998).

An important question that we have not addressed in this work is whether breakup occurs as a progressive buildup of surface energy due to the accumulative interaction with many eddies, or as sharp increments of the surface energy due to interactions with isolated intense turbulent events. Although answering this question necessarily requires temporal analysis, we have shown that the standard deviation of the local surface stretching is much larger than its mean, possibly reflecting inefficient stretching that cancels out. Moreover, at low $W e$, the stretching by outer eddies is mostly balanced by a transfer of energy back to turbulent fluctuations (inner eddies), precluding the buildup of surface energy. These results suggest that the weak turbulent background produces much of this ineffective stretching, whereas the interaction of the drop with just a few intense turbulent structures produces the effective stretching leading to breakup.

\section{Acknowledgements}

A.V.-M. acknowledges the support of the European Research Council COTURB project ERC-2014.AdG-669505. We thank M.P. Encinar for fruitful discussions and comments on the content of the manuscript. 


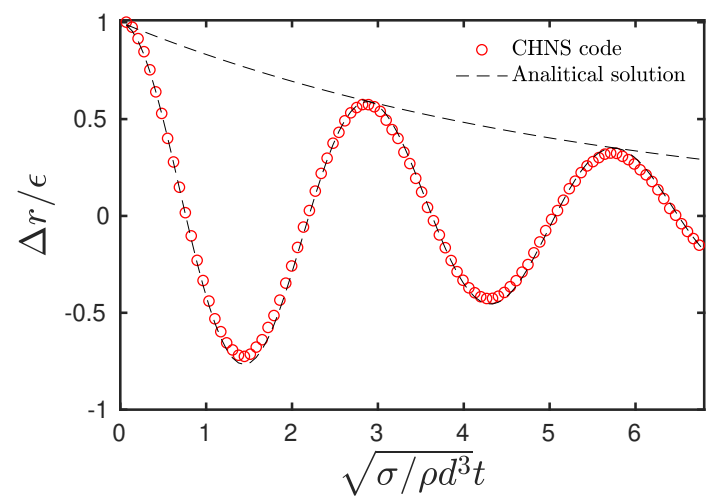

FIgURE 10. Comparison of the analytical solution of $\Delta r / \epsilon$ for a drop with $O h=0.013$ with a numerical simulation with a Cahn-Hilliard-Navier-Stokes (CHNS) code.

\section{Declaration of interest}

The authors report no conflict of interest.

\section{Appendix A. Code validations: oscillations of a viscous drop in a quiescent fluid}

We validate the Cahn-Hilliard-Navier-Stokes code by simulating the oscillations towards equilibrium of a drop in a quiescent fluid. We introduce a drop in a flow without velocity fluctuations, and deform it slightly with an axisymmetric perturbation, so that its evolution towards equilibrium is linear and can be described in terms of axisymmetric spherical harmonics. The solution of this flow was given by Lamb (1924), and reads

$$
r(\theta, t)=r_{0}+\epsilon P_{n}(\cos \theta) \cos \left(f_{n} t\right) \exp \left(-\gamma_{n} t\right),
$$

where $r_{0}=d / 2$ is the radius of the spherical drop, $\epsilon \ll r_{0}$ is the perturbation magnitude, and $P_{n}$ is the Legendre polynomial of order $n$. Here $f_{n}$ and $\gamma_{n}$ are the characteristic oscillation frequency and decay rate of each harmonic,

$$
f_{n}^{2}=\frac{\sigma}{\rho d^{3}} \frac{n(n-1)(n+1)(n+2)}{2 n+1},
$$

and

$$
\gamma_{n}=\frac{\nu}{d^{2}}(n(n-1)+(n+1)(n+2)),
$$

respectively. Here $\sigma$ is the surface tension, $\rho$ and $\rho$ and $\nu$ the density and the kinematic viscosity of the fluid, respectively. The two characteristic time scales of the problem are $\sqrt{\rho d^{3} / \sigma}$ and $d^{2} / \nu$. Their ratio, the Ohnesorge number, $O h=\sqrt{\rho /(\sigma d)} \nu$, is the only parameter of the problem.

We consider a spherical drop with $O h=0.013$, and perturb only its second harmonic, $n=2$. We measure the oscillation of the interface in the direction of the longest semiaxis of the drop $\left(\theta=0\right.$ and $\left.P_{2}=1\right)$, and compare it with the analytical solution. The numerical parameters of the code are the same as those used in the single drop simulations. In figure 10 , we show the evolution of

$$
\Delta r=r(0, t)-r_{0},
$$


in the simulation, and the analytical solution,

$$
\Delta r=\epsilon \cos \left(f_{n} t\right) \exp \left(-\gamma_{n} t\right) .
$$

The match between the two is very good. The characteristic oscillatory frequency given by the code matches the analytical solution with less than $1 \%$ error, whereas the decay rate is reproduced with less than $4 \%$ error.

\section{REFERENCES}

Aiyer, A.K., Yang, D., Chamecki, M. \& Meneveau, C. 2019 A population balance model for large eddy simulation of polydisperse droplet evolution. J. Fluid Mech. 878, 700-739.

Andersson, R. \& Andersson, B. 2006 On the breakup of fluid particles in turbulent flows. AIChE Journal 52, 2020-2030.

Ashurst, W.T., Kerstein, A.R., Kerr, R.M. \& Gibson, C.H. 1987 Alignment of vorticity and scalar gradient with strain rate in simulated navier-stokes turbulence. Phys. Fluids 30, 2343-2353.

Badalassi, V.E., Ceniceros, H.D. \& Banerjee, S. 2003 Computation of multiphase systems with phase field models. J. Comput. Phys. 190, 371-397.

Betchov, R 1956 An inequality concerning the production of vorticity in isotropic turbulence. J. Fluid Mech. 1 (5), 497-504.

Buaria, D., Bodenschatz, E. \& Pumir, A. 2020 Vortex stretching and enstrophy production in high reynolds number turbulence. Phys. Rev. Fluids 5 (10), 104602.

Calabrese, R.V., Chang, T.P.K. \& Dang, P.T. 1986 Drop breakup in turbulent stirred-tank contactors. Part I: Effect of dispersed-phase viscosity. AIChE J. 32, 657-666.

Cardesa, J.I., Vela-Martín, A. \& Jiménez, J. 2017 The turbulent cascade in five dimensions. Science 357, 782-784.

Chen, L.Q. \& Shen, J. 1998 Applications of semi-implicit fourier-spectral method to phase field equations. Comput. Phys. Commun. 108, 147-158.

Coulaloglou, C.A. \& Tavlarides, L.L. 1976 Drop size distributions and coalescence frequencies of liquid-liquid dispersions in flow vessels. AIChE (2), 289-297.

Dodd, M.S. \& Ferrante, A. 2016 On the interaction of taylor length scale size droplets and isotropic turbulence. J. Fluid Mech. 806, 356-412.

Dopazo, C., Lozano, A. \& Barreras, F. 2000 Vorticity constraints on a fluid/fluid interface. Phys. Fluids 12, 1928-1931.

Eastwood, C.D., Armi, L. \& Lasheras, J.C. 2004 The breakup of immiscible fluids in turbulent flows. J. Fluid Mech. 502, 309-333.

Elghobashi, S 2019 Direct numerical simulation of turbulent flows laden with droplets or bubbles. Annu. Rev. Fluid Mech. (0).

Girimaji, S.S. \& Pope, S.B. 1990 Material-element deformation in isotropic turbulence. J. Fluid Mech. 220, 427-458.

HAKANSSON, A. 2019 Emulsion formation by homogenization: Current understanding and future perspectives. Annu. Rev. Food Sci. Technol. 10, 239-258.

HAKANSSON, A. 2020 On the validity of different methods to estimate breakup frequency from single drop experiments. Chem. Engin. Sci. 227, 115908.

Hamlington, P.E., Schumacher, J. \& Dahm, W.J.A. 2008 Local and nonlocal strain rate fields and vorticity alignment in turbulent flows. Phys. Rev. E $\mathbf{7 7}$ (2), 026303.

Hinze, J.O. 1955 Fundamentals of the hydrodynamic mechanism of splitting in dispersion processes. AIChE Journal 1 (3), 289-295.

JACQMin, D. 1999 Calculation of two-phase Navier-Stokes flows using phase-field modeling. $J$. Comput. Phys. 155, 96-127.

Jiménez, J., Wray, A., Saffman, P.G. \& Rogallo, R.S. 1993 The structure of intense vorticity in isotropic turbulence. J. Fluid Mech. 255, 65-90.

Kolmogorov, A.N. 1949 On the disintegration of drops in turbulent flow. In Doklady Akad. Nauk. USSR, , vol. 66, p. 825.

Komrakova, Alexandra E, Eskin, Dmitry \& Derksen, JJ 2015 Numerical study of turbulent liquid-liquid dispersions. AIChE Journal 61 (8), 2618-2633. 
LAmB, H. 1924 Hydrodynamics. University Press.

Lasheras, J.C., Eastwood, C., Martinez-BazÁn, C. \& Montanes, J.L. 2002 A review of statistical models for the break-up of an immiscible fluid immersed into a fully developed turbulent flow. Int. J. Multiph. Flow 28, 247-278.

LiaO, Y. \& LuCAS, D. 2009 A literature review of theoretical models for drop and bubble breakup in turbulent dispersions. Chem. Eng. Sci. 64, 3389-3406.

Luo, H. \& Svendsen, H. 1996 Theoretical model for drop and bubble breakup in turbulent dispersions. AIChE J. 42, 1225-1233.

MaAss S. \& Kraume, M. 2012 Determination of breakage rates using single drop experiments. Chem. Eng. Sci. 70, $146-164$.

Magaletti, F., Picano, F., Chinappi, M., Marino, L. \& Casciola, C. M. 2013 The sharpinterface limit of the Cahn-Hilliard/Navier-Stokes model for binary fluids. J. Fluid Mech. 714, 95-126.

MukherJee, S., Safdari, A., Shardt, O., Kenjereš, S. \& Van den Akker, H.E.A. 2019 Droplet-turbulence interactions and quasi-equilibrium dynamics in turbulent emulsions. J. Fluid Mech. 878, 221-276.

Narsimhan, G., Gupta, J.P. \& Ramkrishna, D. 1979 A model for transitional breakage probability of droplets in agitated lean liquid-liquid dispersions. Chem. Eng. Sci. (2), $257-265$.

Ohkitani, K. \& Kishiba, S. 1995 Nonlocal nature of vortex stretching in an inviscid fluid. Phys. Fluids 7, 411-421.

Perlekar, P. 2019 Kinetic energy spectra and flux in turbulent phase-separating symmetric binary-fluid mixtures. J. Fluid Mech. pp. 459-474.

Perlekar, P., Biferale, L., Sbragaglia, M., Srivastava, S. \& Toschi, F. 2012 Droplet size distribution in homogeneous isotropic turbulence. Phys. Fluids (6), 065101.

Qian, D., Mclaughlin, J. B., Sankaranarayanan, K., Sundaresan, S. \& Kontomaris, K. 2006 Simulation of bubble breakup dynamics in homogeneous turbulence. Chem. Eng. Commun. 193 (8), 1038-1063.

RALlison, J.M. 1984 The deformation of small viscous drops and bubbles in shear flows. Annu. Rev. Fluid Mech. 16, 45-66.

RAmkrishna, D. \& Singh, M.R. 2014 Population balance modeling: current status and future prospects. Annu. Rev. Chem. Biomol. Eng. pp. 123-146.

Risso, F. \& FABRE, J. 1998 Oscillations and breakup of a bubble immersed in a turbulent field. J. Fluid Mech. 372, 323-355.

Roccon, A., De Paoli, M., Zonta, F. \& Soldati, A. 2017 Viscosity-modulated breakup and coalescence of large drops in bounded turbulence. Phys. Rev. Fluids 2 (8), 083603.

Rosti, M.E., Ge, Z., Jain, S.S., Dodd, M.S. \& Brandt, L. 2019 Droplets in homogeneous shear turbulence. J. of Fluid Mech. pp. 962-984.

Scarbolo, L., Bianco, F. \& Soldati, A. 2015 Coalescence and breakup of large droplets in turbulent channel flow. Phys. Fluids 27, 073302.

ShaO, C., Luo, K., YAng, Y. \& FAn, J. 2018 Direct numerical simulation of droplet breakup in homogeneous isotropic turbulence: The effect of the weber number. Int. J. Multiph. Flow 107, 263-274.

Soligo, G., Roccon, A. \& Soldati, A. 2019 Mass-conservation-improved phase field methods for turbulent multiphase flow simulation. Acta Mechanica 230, 683-696.

Soligo, G., Roccon, A. \& Soldati, A. 2020 Effect of surfactant-laden droplets on turbulent flow topology. Phys. Rev. Fluids 5, 073606.

Solsvik, J. \& Jakobsen, H.A. 2015 Single drop breakup experiments in stirred liquid-liquid tank. Chem. Eng. Sci. 131, 219-234.

Yi, Lei, Toschi, Federico \& Sun, Chao 2021 Global and local statistics in turbulent emulsions. Journal of Fluid Mechanics 912.

Yue, P., Zhou, C. \& Feng, J. J. 2007 Spontaneous shrinkage of drops and mass conservation in phase-field simulations. J. Comput. Phys. 223, 1-9.

Zhang, Yujie \& Ye, Wenjing 2017 A flux-corrected phase-field method for surface diffusion. Commun. Comput. Phys. 22, 422-440.

Zhong, Cheng \& Komrakova, Alexandra 2019 Liquid drop breakup in homogeneous isotropic turbulence. Int. J. Numer. Method H. . 